\title{
ZHX2 enhances the cytotoxicity of chemotherapeutic drugs in liver tumor cells by repressing MDR1 via interfering with NF-YA
}

\author{
Hongxin $\mathrm{Ma}^{1,}{ }^{*}$, Xuetian Yue ${ }^{1, *}$, Lifen $\mathrm{GaO}^{1}{ }^{1}$, Xiaohong Liang ${ }^{1}$, Wenjiang Yan ${ }^{1}$, Zhenyu \\ Zhang $^{1}$, Haixia Shan ${ }^{1}$, Hualin Zhang ${ }^{1}$, Brett T. Spear ${ }^{2}$ and Chunhong Ma ${ }^{1}$ \\ ${ }^{1}$ Key Laboratory for Experimental Teratology of Ministry of Education and Department of Immunology, Shandong University \\ School of Medicine, Jinan, Shandong, P.R. China \\ ${ }^{2}$ Department of Microbiology, Immunology \& Molecular Genetics and Markey Cancer Center, University of Kentucky College \\ of Medicine, Lexington, KY, USA \\ * These authors contributed equally to the work
}

Correspondence to: Chunhong Ma, email: machunhong@sdu.edu.cn

Keywords: ZHX2, transcriptional repression, chemoresistance, p-glycoprotein, apoptosis

Received: September 23, $2014 \quad$ Accepted: November 25, $2014 \quad$ Published: November 26, 2014

This is an open-access article distributed under the terms of the Creative Commons Attribution License, which permits unrestricted use, distribution, and reproduction in any medium, provided the original author and source are credited.

\section{ABSTRACT}

We previously reported the tumor suppressor function of Zinc-fingers and homeoboxes 2 (ZHX2) in hepatocellular carcinoma (HCC). Other studies indicate the association of increased ZHX2 expression with improved response to high dose chemotherapy in multiple myeloma. Here, we aim to test whether increased ZHX2 levels in HCC cells repress multidrug resistance 1(MDR1) expression resulting in increased sensitivity to chemotherapeutic drugs. We showed evidence that increased ZHX2 levels correlated with reduced MDR1 expression and enhanced the cytotoxicity of CDDP and ADM in different HCC cell lines. Consistently, elevated ZHX2 significantly reduced ADM efflux in HepG2 cells and greatly increased the CDDP-mediated suppression of liver tumor growth in vivo. Furthermore, immunohistochemical staining demonstrated the inverse correlation of ZHX2 and MDR1 expression in HCC tissues. Luciferase report assay showed that ZHX2 repressed the MDR1 promoter activity, while knockdown of NF-YA or mutating the NF-Y binding site eliminated this ZHX2mediated repression of MDR1 transcription. Co-IP and ChIP assay further suggested that ZHX2 interacted with NF-YA and reduced NF-Y binding to the MDR1 promoter. Taken together, we clarify that ZHX2 represses NF-Y-mediated activation of MDR1 transcription and, in doing so, enhances the effects of chemotherapeutics in HCC cells both in vitro and in vivo.

\section{INTRODUCTION}

Hepatocellular carcinoma (HCC) is one of the most common cancers worldwide with high mortality, and poor prognosis. Although a large number of therapeutic agents have been evaluated for the treatment of HCC, most have been ineffective due to the high chemoresistance, especially the multidrug resistance (MDR) of liver cancer cells [1, 2]. Overexpression of multidrug resistance protein 1(MDR1, also known as p-glycoprotein), an ATPdependent pump, causes the efflux of various hydrophobic compounds and xenobiotics leading to MDR [3]. Accumulated data confirm the important role of MDR1 in the resistance of HCC cells against different chemodrugs, including the commonly used chemotherapeutic drugs cisplatin [cis-diamminedichloroplatinum (CDDP)] and Adriamycin (ADM)[4-6] . Strategies targeting MDR1 have been recognized as a potential method to restore chemotherapeutic sensitivity of cancer cells, however, strategies have had minimal clinical benefit $[3,7]$. Therefore, understanding the regulation of MDR1 expression in $\mathrm{HCC}$ is essential to develop more effective treatments.

Previous studies have identified a number of transcription factors binding sites in $M D R 1$ promoter. A conserved CCAAT element (Y-box), located between -82 to -73 in the human MDR1 promoter is absolutely required for basal and inducible expression of the human 
Table 1: Immunohistochemical stainning of ZHX2 and MDR1 expression in clinical specimens.

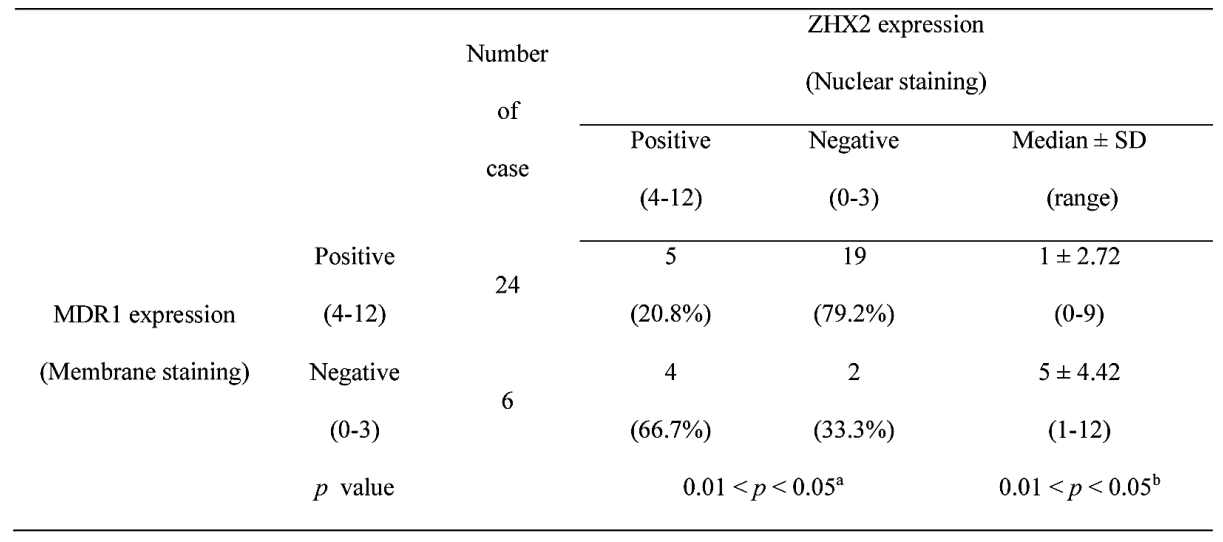

${ }^{a} p$ values were obtained from the Chi-square test.

${ }^{\mathrm{b}} p$ values were obtained from the non-parametric test.

MDR1 gene $[8,9]$.The nuclear protein NF-Y, a complex consisting of A, B, and $\mathrm{C}$ subunits, recognizes the $Y$-box sequences and orchestrates $M D R 1$ promoter activation [9, 10]. The identification of NF-Y as a central mediator of MDR1 activation makes it an attractive molecular target for manipulating the MDR phenotype and therapeutic intervention.

The Zinc-Fingers and Homeoboxes 2 (ZHX2) gene is a member of a small gene family that also includes $Z H X 1$ and $Z H X 3$ [11]. Two-hybrid studies indicate that ZHX2 can form homodimers as well as heterodimers with other ZHX family members and with NF-YA [12]. Consistent with these data, ZHX2 regulates the NF-YAdependent genes $c d c 25 C$ and Hexokinase II (HKII) and has been implicated in cell cycle control $[12,13]$. A potential role for ZHX2 in HCC came from studies showing that ZHX2 represses expression of alpha-fetoprotein (AFP), glypican-3 (GPC3) and H19, three genes that are frequently activated in $\mathrm{HCC}[14,15]$. We recently demonstrated that ZHX2 overexpression leads to G1 arrest and down-regulation of cyclin $\mathrm{A}$ and cyclin $\mathrm{E}$ expression in HCC cell lines [16]. Altered ZHX2 expression has been reported in hepatic and hematological malignancies [1719]. Interestingly, high ZHX2 expression is significantly associated with an improved response and longer survival after high dose-chemotherapy in patients with multiple myeloma [17], suggesting that ZHX2 might influence drug resistance in cancer cells.

Based on the function of ZHX2 as a transcriptional repressor and its known interaction with NF-YA, we hypothesized that ZHX2 might inhibit MDR1 expression in liver cancer cells, resulting in reduced efflux of chemotherapeutic drugs and subsequent increased sensitivity to these agents. To test this, the correlation of ZHX2 and MDR1 expression was evaluated in HCC tissues. Then ZHX2 levels were experimentally increased or decreased in several liver cancer cell lines, followed by treatment with CDDP and ADM. Our results indicate that higher ZHX2 levels reduced MDR1 expression and decreased drugs efflux in all HCC lines tested. Consistently, ZHX2 significantly enhanced the sensitivity of $\mathrm{HCC}$ cells to chemotherapeutic drugs both in vitro and in vivo. Taken together, our data identify MDR1 as a new target of the tumor suppressor ZHX2 and suggest that ZHX2 maybe a novel target for the treatment of liver cancer.

\section{RESULTS}

\section{The expression level of $\mathrm{ZHX2}$ in $\mathrm{HCC}$ tissues negatively correlates with that of MDR1}

We first evaluated the correlation of ZHX2 and MDR1 expression in HCC tissues. To address that, thirty HCC samples were involved to do immunohistochemical staining with antibodies against ZHX2 and MDR1. Consistent with our previous study [16], nucleic ZHX2 could be detected in less than 35\% (9/30) involved HCC cases (Table 1). Moreover, MDR1 expression in HCC tissue sections with nucleic ZHX2 was comparatively lower than that in HCC tissue sections without nucleic ZHX2 (Figure 1). Analysis results of Chi-square test and non-parametric test further confirmed the reverse correlation of nucleic ZHX2 with MDR1 in HCC (Table 1 ). Both the positive percentage (score of 4-12) and the expression intensity of nuclear ZHX2 (displayed as median $\pm \mathrm{SD}$ ) were significantly lower in MDR1-positive staining samples (score of 4-12) than that in MDR1negative staining samples $(p<0.05)$. These indicated that reduced nuclear ZHX2 level might be responsible for enhanced MDR1 expression in HCC. 


\section{ZHX2 decreases MDR1 expression and reduces drug efflux from HCC cells}

In order to further confirm the negative regulation of ZHX2 on MDR1 in HCC, we then did in vitro studies. ZHX2 and MDR1 mRNA levels were compared in several liver cancer cell lines. RT-PCR analysis showed an inverse correlation between MDR1 and ZHX2 expression: cells with higher MDR1 mRNA levels (HepG2 and HepG2.2.15 cells) had lower $Z H X 2$ mRNA levels whereas those with lower MDR1 (SMMC7721 cells) had higher ZHX2 (Figure S1A). Interestingly, ZHX2 expression level correlated with CDDP sensitivity in HCC cells (Figure S1B), indicating that ZHX2 closely correlates with MDR1 expression and chemotherapy sensitivity of $\mathrm{HCC}$ cells. To explore further the relationship between these two genes, ZHX2 was overexpressed or knocked down by transient transfection. As shown in Figure 2A, ZHX2 overexpression led to decreased MDR1 mRNA levels in HepG2 and HepG2.2.15 cells, whereas ZHX2 knockdown with two different siRNAs (ZHX2-1674, ZHX2-2360) resulted in elevated MDR1 mRNA levels in SMMC7721 cells. This difference was also seen at the protein level as determined by western blot (Figure 2B and Figure S2). These data support the possibility that ZHX2 represses MDR1 expression in HCC cells.

MDR1 is a well-known ATP-dependent drug efflux pump. To evaluate the effect of ZHX2 on regulating the MDR1 transporter activity, HepG2 cells were transfected with pEGFP-ZHX2 and then treated with ADM, which emits a natural red fluorescence. EGFP-ZHX2 expression and ADM autofluorescence intensity were detected by fluorescence microscopy. As shown in Figure 2C, red fluorescence was higher in EGFP-ZHX2 expressing cells than untransfected cells after ADM treatment, indicating greater ADM accumulation in EGFP-ZHX2 transfected cells. Enhanced ADM accumulation in EGFP-ZHX2 expressing cells was further confirmed by flow cytometry. The red MFI in EGFP-positive cells was significantly higher than that in EGFP-negative cells 4 hours after ADM treatment (Figure 2D, left panel). The red MFI in EGFP-positive cells remained higher than EGFP-negative cells 2 hours after ADM withdraw (Figure 2D, right panel), suggesting enhanced ADM retention in EGFPZHX2 overexpressing cells. Consistently, EGFP-ZHX2 positive cells exhibited a decreased ADM releasing index compared with EGFP-ZHX2 negative cells (Figure 2E). Taken together, these data suggest that ZHX2 suppresses MDR1 expression and decreases drug efflux, resulting in increased intracellular ADM levels.
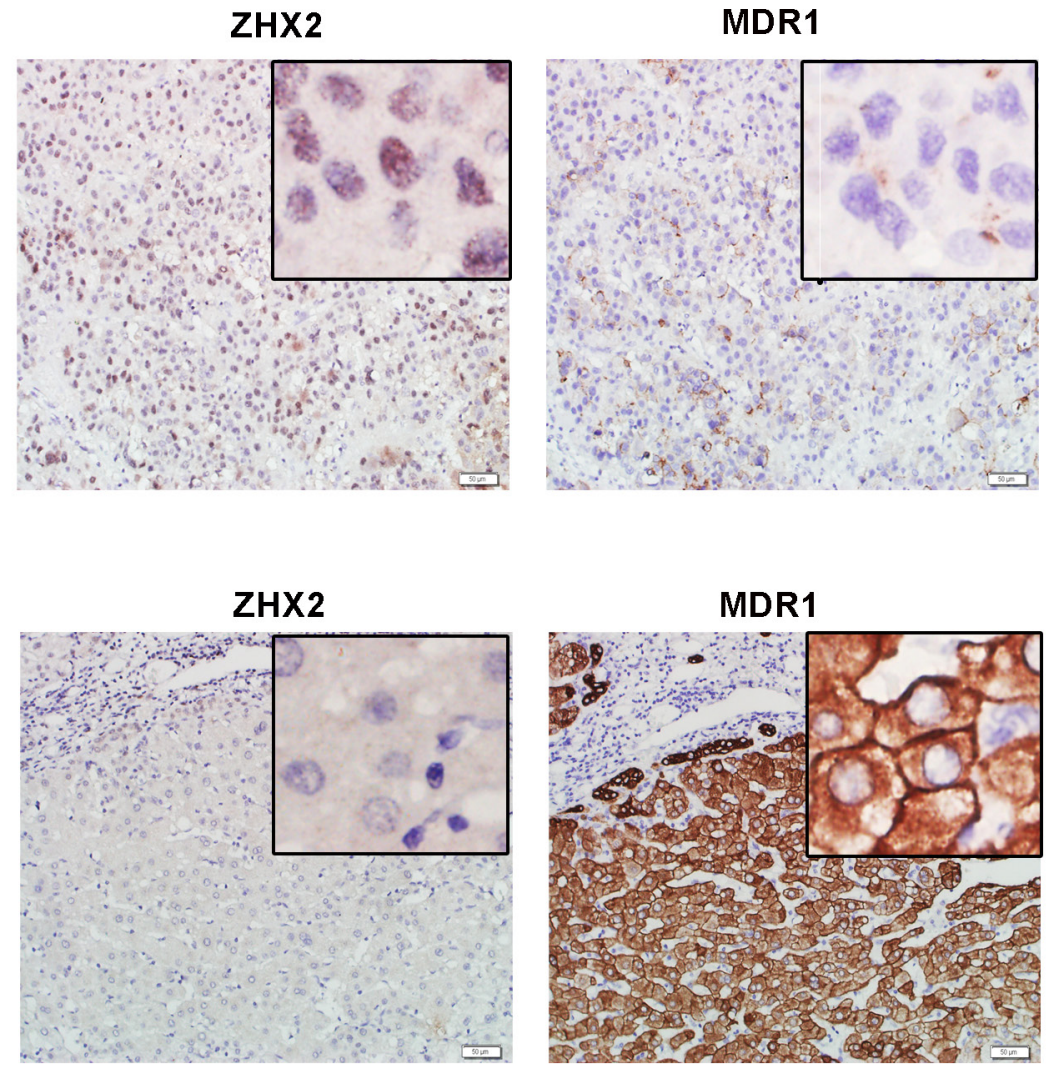

Figure 1: ZHX2 expression is inverse correlated to the expression of MDR1 in HCC. Immunohistochemical staining of ZHX2 and MDR1 in adjacent sections of cancer biopsies from 2 of the $30 \mathrm{HCC}$ samples (upper panels, ZHX2 high and MDR1 low ; lower panels, ZHX2 low and MDR1 high). bars $=50 \mu \mathrm{m}$; statistical results were shown in Table 1. 
A

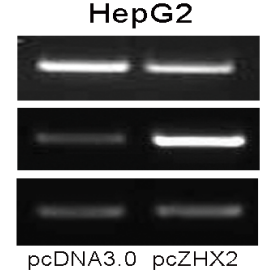

B

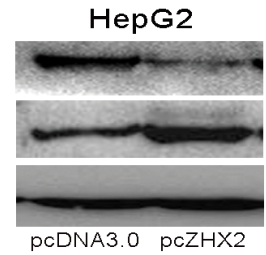

HepG2.2.15

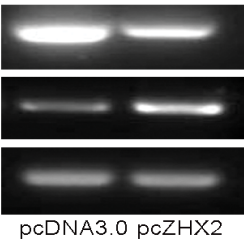

HepG2.2.15

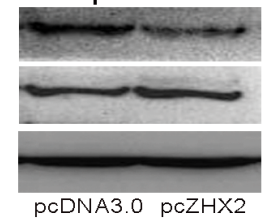

SMMC7721

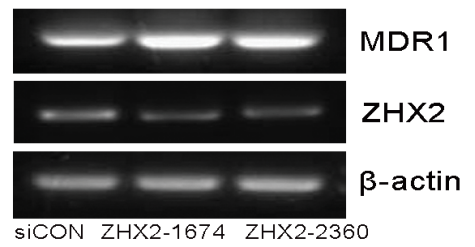

SMMC7721

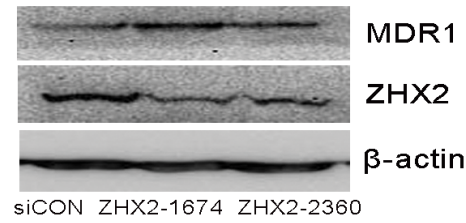

C HepG2
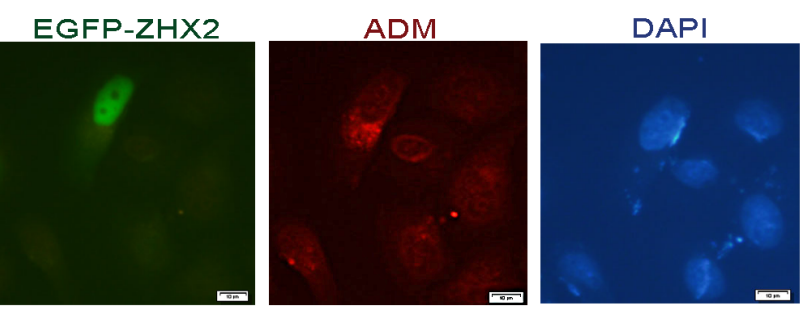

D
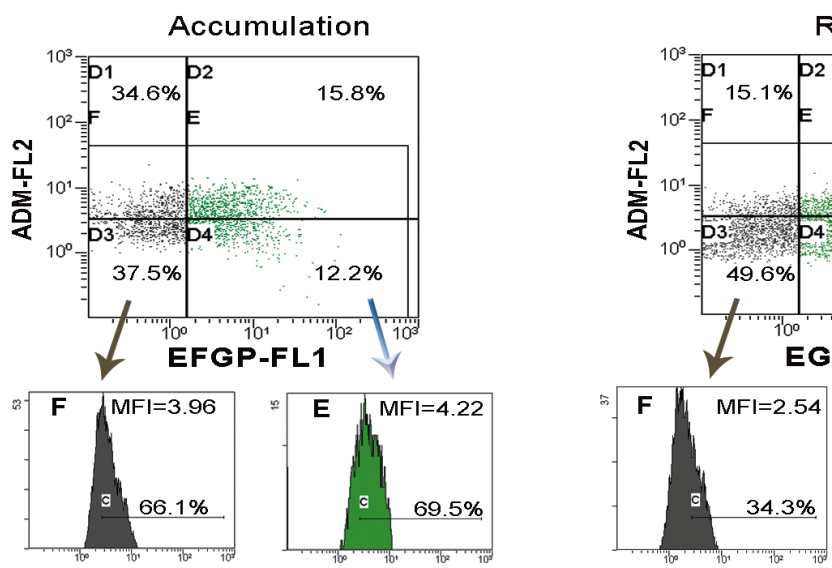

Retention

\section{E}

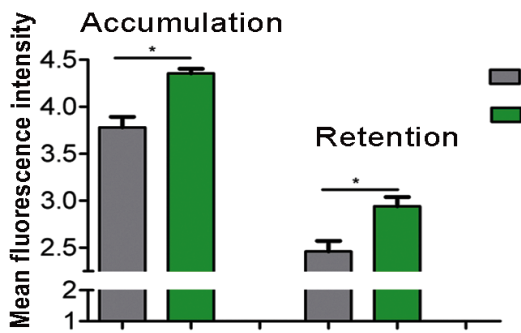

Releasing index
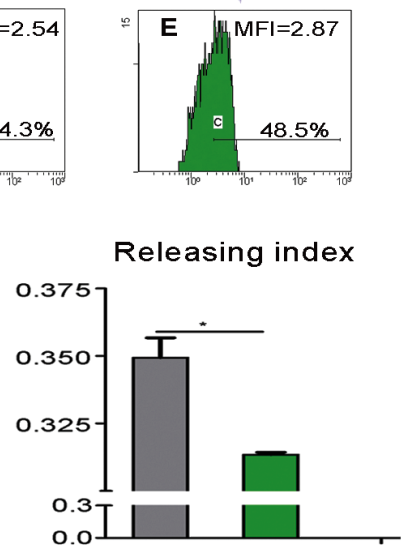

Figure 2: ZHX2 suppresses MDR1 expression and increases ADM retention of HCC cells. (A and B) $Z H X 2, M D R 1$ and $\beta$-actin mRNA levels(A) and protein levels (B) were determined by RT-PCR and western blot in HepG2 and HepG2.2.15 cells transfected with pcDNA3.0 or pcZHX2 and in SMMC7721 cells that were transfected with ZHX2 siRNAs or control siRNA (siCON). (C) ZHX2EGFP expression and intracellular ADM were determined by fluorescence microscopy of HepG2 cells in pEGFP-ZHX2-transfected cells treated with ADM as described in Methods and Materials. Representative panels are shown. Red, autofluorescence of ADM ; Green, fluorescence of EGFP-ZHX2 ; Blue, DAPI staining. Bars, $10 \mu \mathrm{m}$. (D) ADM accumulation (left panels) and retention (right panels) in pEGFP-ZHX2 transfected HepG2 cells were analyzed by flow cytometry. (E) Left panel: ADM accumulation and retention based on data in Figure 2D. Right panel: ADM-releasing index was calculated based on accumulation and retention data from flow cytometry. Data are shown as the mean $\pm \mathrm{SD}(\mathrm{n}=3) ;{ }^{*} p<0.05$. 

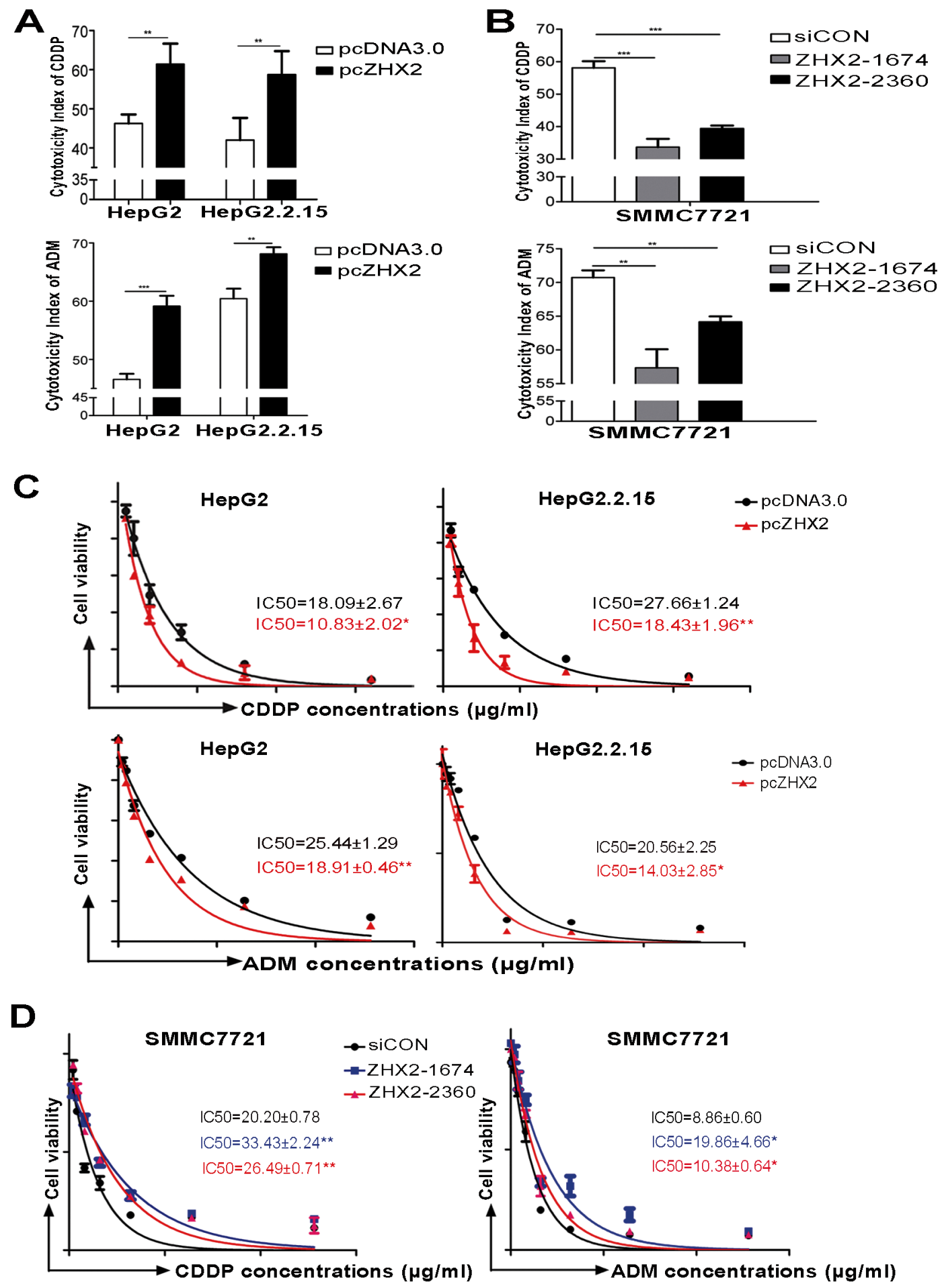

Figure 3: Higher ZHX2 levels increase the sensitivity of HCC cells to the cytotoxic effects of CDDP and ADM. HepG2 and HepG2.2.15 cells were transfected with pcDNA3.0 or pcZHX2 (A), whereas SMMC7721 cells were transfected with siCON or ZHX2siRNAs (B). After 24 hours, cells were treated with CDDP (upper panels) or ADM (lower panels) and cultured for another 24 hours. The cytotoxicity was calculated as described in Materials and Methods. Data are shown as the mean $\pm \mathrm{SD}(\mathrm{n}=4) ; * * p<0.01, * * * p<0.001$. (C and $\mathrm{D}$ ) $\mathrm{IC}_{50}$ of CDDP (upper panels) or ADM (lower panels) in cell lines transfected as described above in $\mathrm{A}$ and $\mathrm{B}$. The $\mathrm{IC}_{50}$ was calculated as described in Materials and Methods. Data are shown as the mean $\pm \mathrm{SD}(\mathrm{n}=3) ;{ }^{*} p<0.05,{ }^{* *} p<0.01$. 


\section{Higher ZHX2 expression increases the cytotoxicity of chemotherapeutic drugs}

The ability of ZHX2 to repress MDR1 led us to consider whether elevated ZHX2 levels would increase drug sensitivity in $\mathrm{HCC}$ cells. To test this, the cytotoxicity index of CDDP or ADM was determined in ZHX2overexpressing cells or ZHX2-knockdown cells. In ZHX2overexpressing cell lines (HepG2 and HepG2.2.15), the cytotoxicity index increased significantly after treatment with both CDDP and ADM (Figure 3A) compared to pcDNA3.0-transfected cells treated with these drugs. In accordance, knock-down of ZHX2 in SMMC7721 cells decreased the cytotoxicity index of both CDDP and $\mathrm{ADM}$ (Figure 3B). This is further supported by $\mathrm{IC}_{50}$ assay measured with increasing amounts of CDDP or ADM in different cell populations (Figure $3 \mathrm{C}$ and $\mathrm{D}$ ). These data indicate that increased ZHX2 levels result in increased sensitivity of HCC cells to these chemotherapeutic drugs.

\section{ZHX2 increases CDDP-induced apoptosis in HepG2 cells}

Since CDDP acts to increase apoptosis by DNA cross-linking, we next tested whether elevated ZHX2 would enhance CDDP-mediated DNA damage and apoptosis in HepG2 cells as judged by chromatin condensation and nuclear fragmentation [20]. ZHX2 overexpression by itself did not increase the number of apoptotic, sub-G1 cells compared to pcDNA3.0 transfected cells (Figure 4A). As expected, a distinct sub-G1 peak was detected in CDDP-treated HepG2 cells. Interestingly, ZHX2 overexpression significantly increased the sub-G1 population after CDDP treatment, indicating that elevated ZHX2 enhances CDDP-induced apoptosis. This increased apoptosis was further verified by Hoechst 33258 and DAPI staining in HepG2 cells, which showed increased chromatin condensation (Figure 4B) and flow cytometry after staining with Annexin $\mathrm{V}$ and propidium iodide (PI, Figure 4C).

To further analyze the effect of ZHX2 on CDDPinduced apoptosis, several apoptosis-related proteins were monitored by western blot. Consistent with earlier studies , CDDP treatment increased levels of cytoplasmic Cytochrome C, cleaved caspase-3, cleaved caspase-9 and cleaved PARP (Figure 4D). Moreover, although ZHX2 overexpression did not alter overall levels of these proteins, pcZHX2 transfection enhanced the CDDPinduced levels of apoptotic products (Figure 4D). This was particularly true for caspase- 9 , since nearly twice the levels of cleaved caspase-9 were detected in HepG2 cells transfected with ZHX2 and treated with CDDP compared with cells treated with CDDP alone (Figure $4 \mathrm{D}$, right panel). Collectively, these results indicate that ZHX2 overexpression enhanced CDDP cytotoxicity by increasing drug-induced apoptosis in $\mathrm{HepG} 2$ cells.

\section{ZHX2 overexpression enhances CDDP-mediated inhibition of HCC growth in nude mice}

In order to detect whether ZHX2 and CDDP could cooperate to inhibit tumor growth in vivo, HepG2.2.15 xenograft tumors were treated with CDDP and/or plasmid DNA (pcDNA3.0 or pcZHX2). As seen in previous studies [16], either CDDP treatment or ZHX2 overexpression alone inhibited xenograft growth significantly (Figure $5 \mathrm{~A})$. However, the combination of pcZHX2 and CDDP cooperatively inhibited tumor growth to a greater extent than treatment with pcZHX2 or CDDP alone (Figure $5 \mathrm{~A})$. Consistent with the diminished growth curves, tumor weights at time of sacrifice were also significantly reduced in mice with combined treatment compared to mice treated with pcZHX2 or CDDP alone (Figure 5B). Immunohistochemistry staining verified the ZHX2 overexpression in tumors injected with pcZHX2 (Figure 5C). TUNEL staining confirmed that ZHX2 promoted CDDP-induced apoptosis of tumor cells (Figure 5D). These results indicated that ZHX2 overexpression enhances the ability of CDDP to inhibit HepG2.2.15 growth in vivo.

\section{ZHX2-mediated repression on MDR1 promoter activity requires NF-Y}

Previous studies indicate that ZHX2 functions as a transcriptional repressor $[9,12,13]$. We therefore tested whether ZHX2 represses MDR1 promoter activity. Results of cotransfection and luciferase assays showed that ZHX2 overexpression significantly decreased MDR1 promoter activity in HepG2 and HepG2.2.15 cells (Figure 6A). Conversely, ZHX2 knock-down by siRNAs greatly enhanced the MDR1 promoter activity in SMMC7721 cells (Figure 6B). The decrease and increase in luciferase levels showed a dose-dependent response in cells that were cotransfected with ZHX2 expression vectors or ZHX2 siRNAs, respectively (Figure 6A and B).

Previous studies identified a NF-YA binding ATTGG element (known as $Y$ box) in the MDR1 promoter that is important for full MDR1 promoter activity [9]. Furthermore, ZHX2 was shown to interact with the NFYA subunit and inhibit the ability of NF-Y to transactivate target genes [9]. To examine whether ZHX2 regulates MDR1 promoter activity via the $Y$ box in HepG2 cells, ZHX2 cotransfections were performed with wild-type promoter pGL3-Mp or the pGL3-mMp in which the $A T T G G$ motif was mutated (Figure 6C). In contrast to pGL3-Mp, the mutant promoter pGL3-mMp was not repressed by ZHX2 (Figure 6D). Furthermore, ZHX2mediated repression of the $M D R 1$ promoter was dependent on NF-Y since this repression was no longer evident when 
A

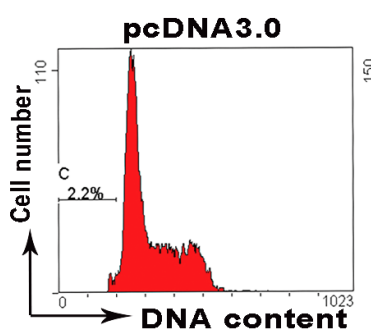

B
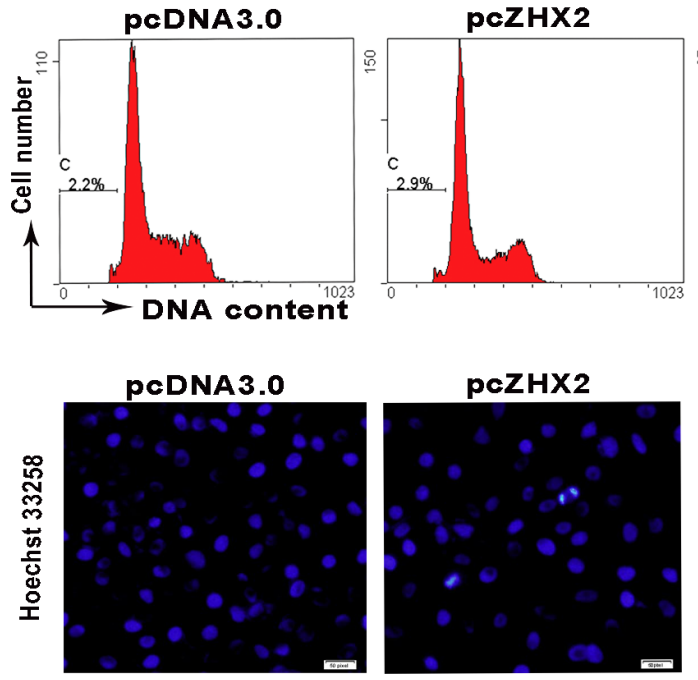

pcZHX2

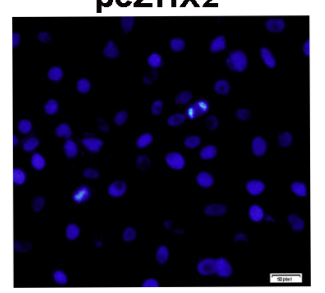

PCDNA3.0+CDDP
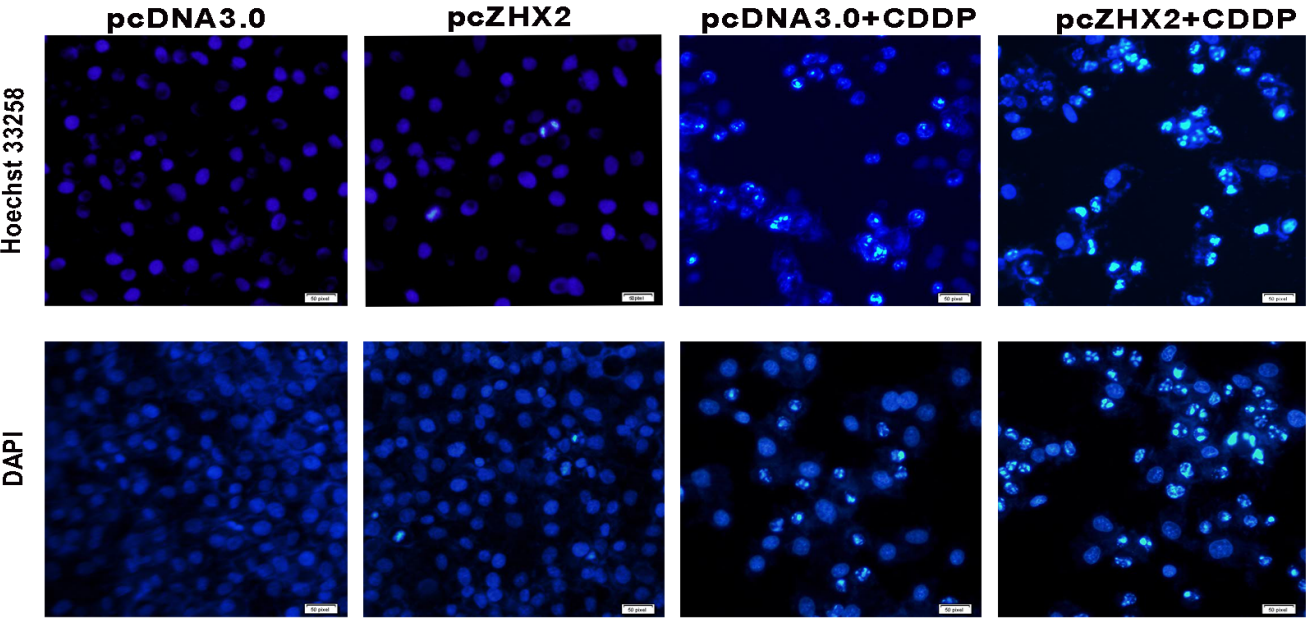

C
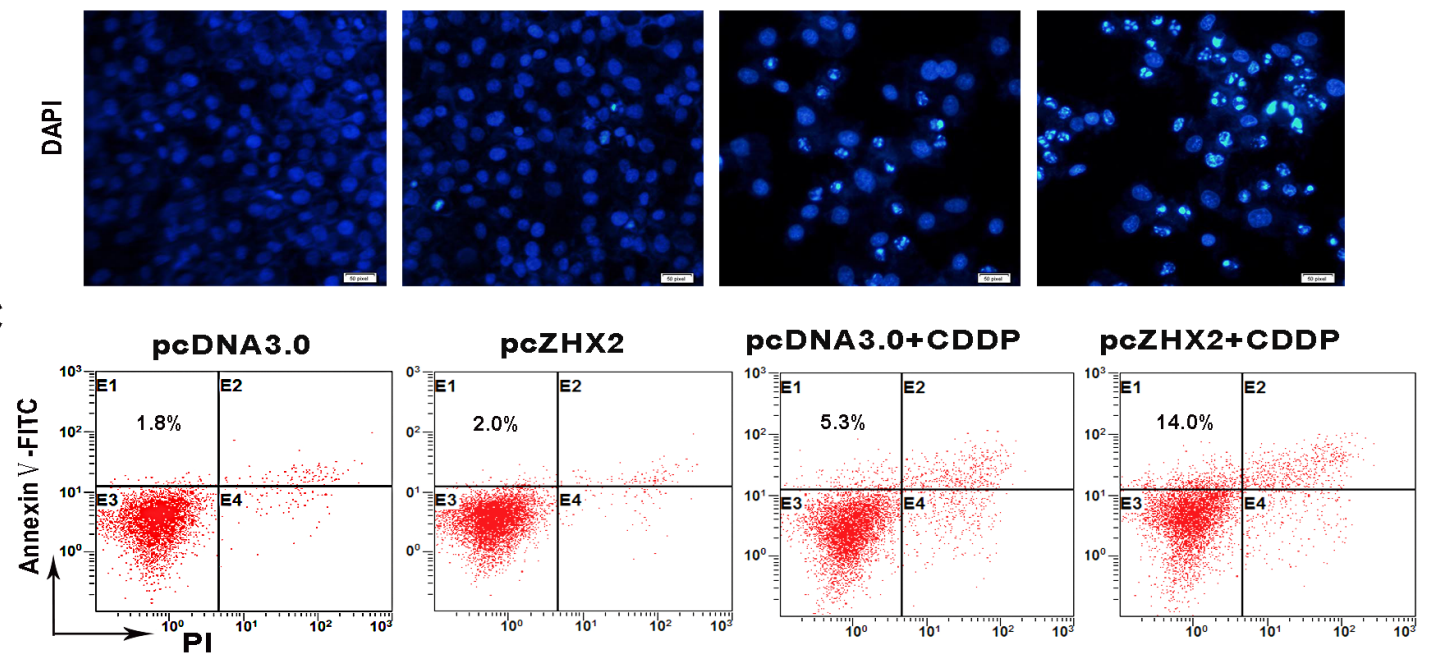

D
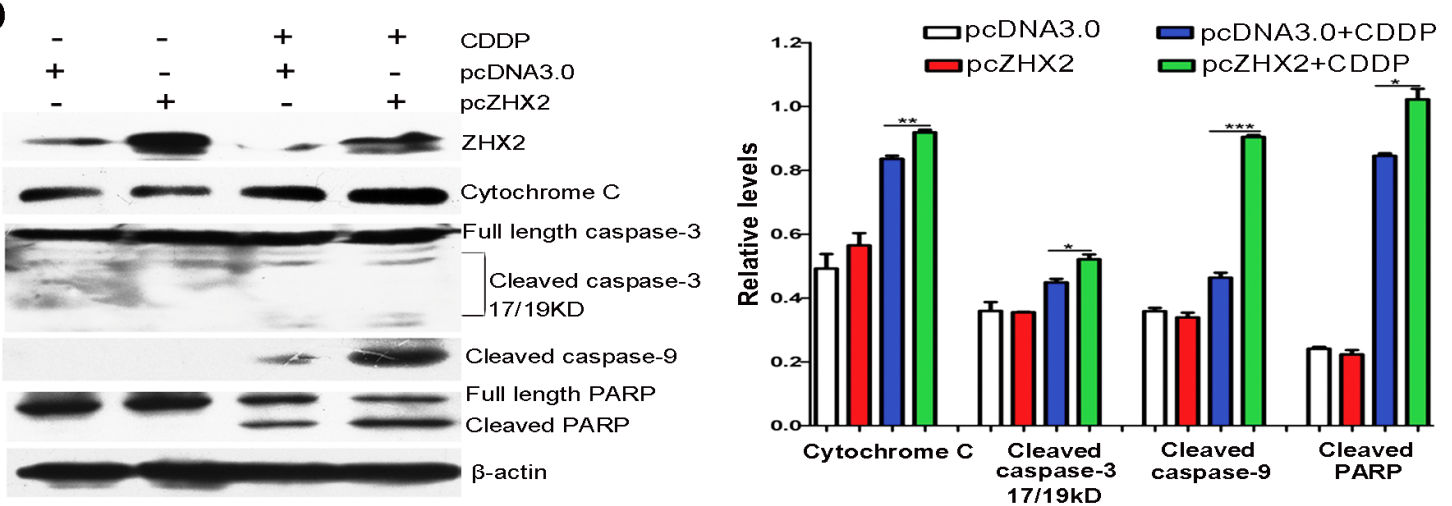

Figure 4: Increased ZHX2 levels in HepG2 cells enhance CDDP-induced apoptosis and activate the caspase pathway. HepG2 cells transfected with pcDNA3.0 or pcZHX2 were treated with CDDP for 24 hours. (A) Cells were incubated with PI and analyzed by flow cytometry. The percentage of cells with fragmented DNA (sub-G1 peak) are shown. (B) Staining of cells with Hoechst 33258 (upper images) and DAPI (lower images). Bars, 50 pixel . (C) Flow cytometry after staining with PI and Annexin V to determine the percentage of Annexin V-stained cells. (D) Western blot analysis to determine levels of ZHX2, Cytochrome C, full-length and cleaved caspase 3, cleaved caspase 9, full-length and cleaved PARP, and $\beta$-actin. Western blot data from three independent experiments are quantitated in the right panel, with levels if indicated protein shown relative to $\beta$-actin. ${ }^{*} p<0.05,{ }^{* *} p<0.01,{ }^{* * *} p<0.001$. 
NF-YA was knocked down by siRNA (Figure 6E).

The transfection data described above led us to consider whether $\mathrm{ZHX} 2$ bound directly to the MDR1 promoter or inhibited NF-Y activity by an indirect mechanism. As previous report in HEK-293, Co-IP demonstrated the interaction of $\mathrm{ZHX} 2$ and NF-YA proteins in HepG2 cells (Figure 7A) [9]. To directly test whether ZHX2 bound to the MDR1 promoter, ChIP were performed using HepG2 cells transfected with pcZHX2HA or pcEGFP-HA and oligonucleotides specific for the $M D R 1$ promoter (Figure 6C). As shown in Figure 7B, a specific PCR amplification was detected in the anti-HA immunoprecipitation from HepG2 cells transfected with
pcZHX2-HA but not in pcEGFP-HA, indicating that $\mathrm{ZHX} 2$ bound to the MDR1 promoter. Interestingly, a relative weak PCR amplification was detected in the antiNF-YA immunoprecipitation from HepG2 cells transfected with pcZHX2-HA than that in pcEGFP-HA (Figure 7 $B$ and $C$ ), suggested that the presence of transfected ZHX2 could influence the NF-YA binding to the MDR1 promoter. Taken together, these data suggest that ZHX2 interacts directly with NF-Y on the MDR1 promoter and that this interaction inhibits NF-Y-mediated activation of MDR1 transcription.
A

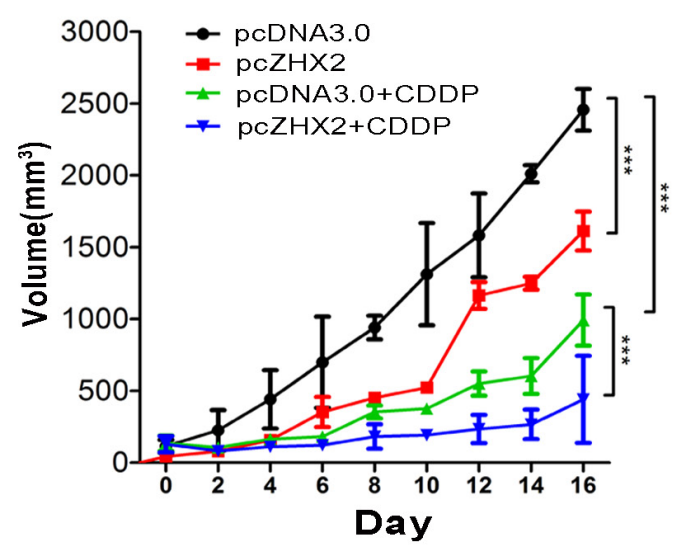

C
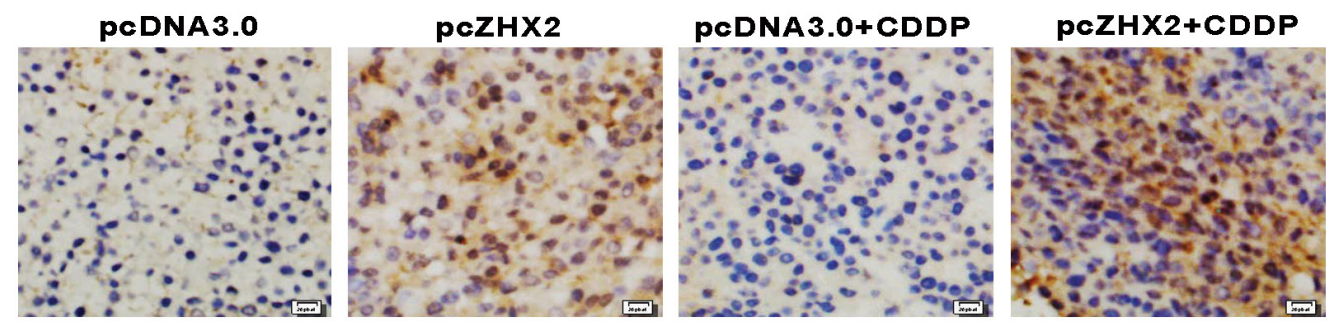

D

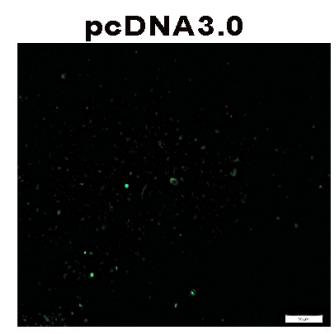

PcZHX2

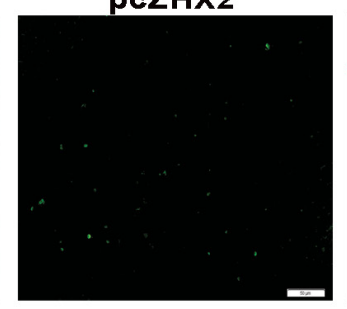

B

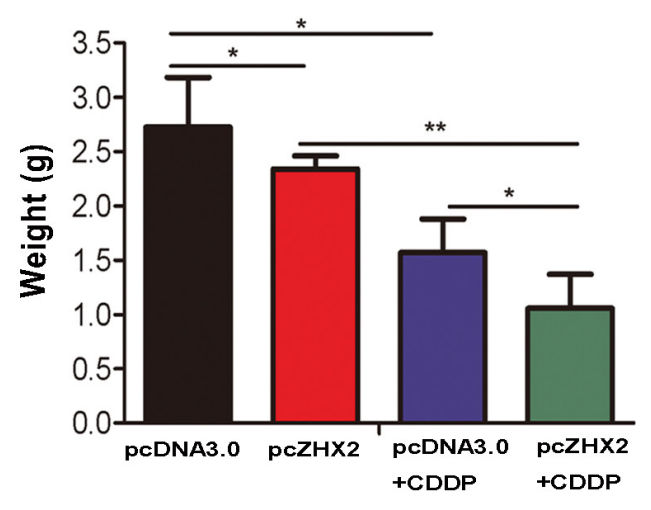

Figure 5: ZHX2 combined with CDDP act cooperatively to inhibit xenograft tumor growth in nude mice. Nude mice containing subcutaneous HepG2.2.15 tumors were randomized to receive intra-tumor injections of pcDNA3.0 or pcZHX2, and half of each group were also injected with CDDP. (A) Tumor volume was calculated every other day for the entire 16-day study. Data are shown as the mean \pm SD ( Two-way ANOVA, $* * * p<0.001$ ). (B) Tumor weights in four different cohorts were measured at the end of the experiment. Data are shown as the mean $\pm \mathrm{SD}(\mathrm{n} \geq 4) ;{ }^{*} p<0.05,{ }^{*} p<0.01$. (C) Immunohistochemical analysis of ZHX2 expression in tumor samples. Bars, 20 pixel. (D) TUNEL staining as a measure of apoptosis in tumor samples indicate greatest staining in pcZHX2 plus CDDP injected tumors. Bars, $50 \mu \mathrm{m}$. 
A
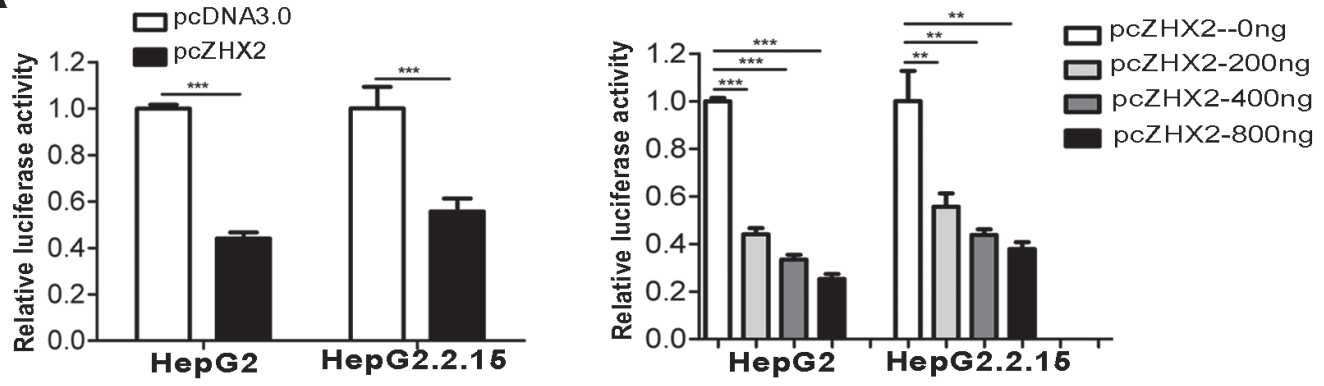

B
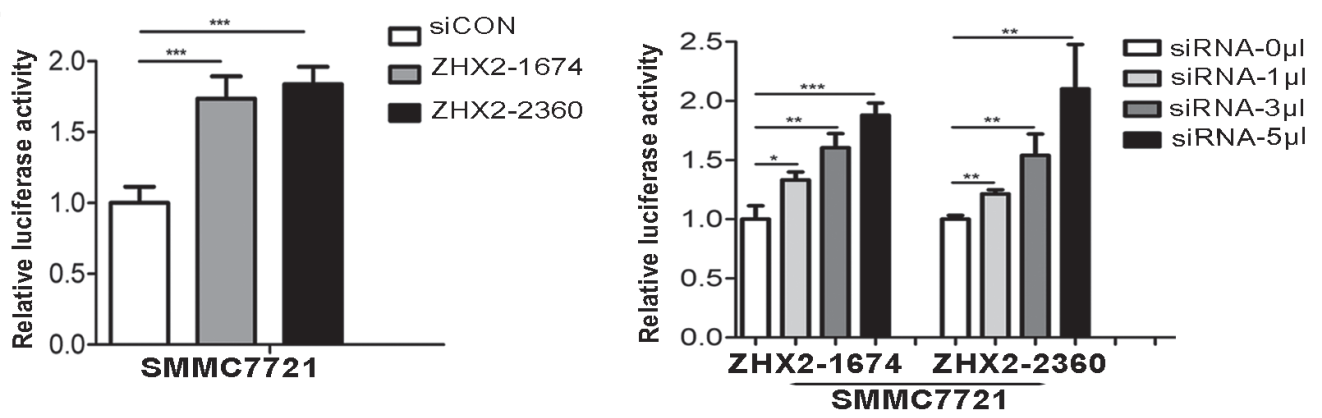

C
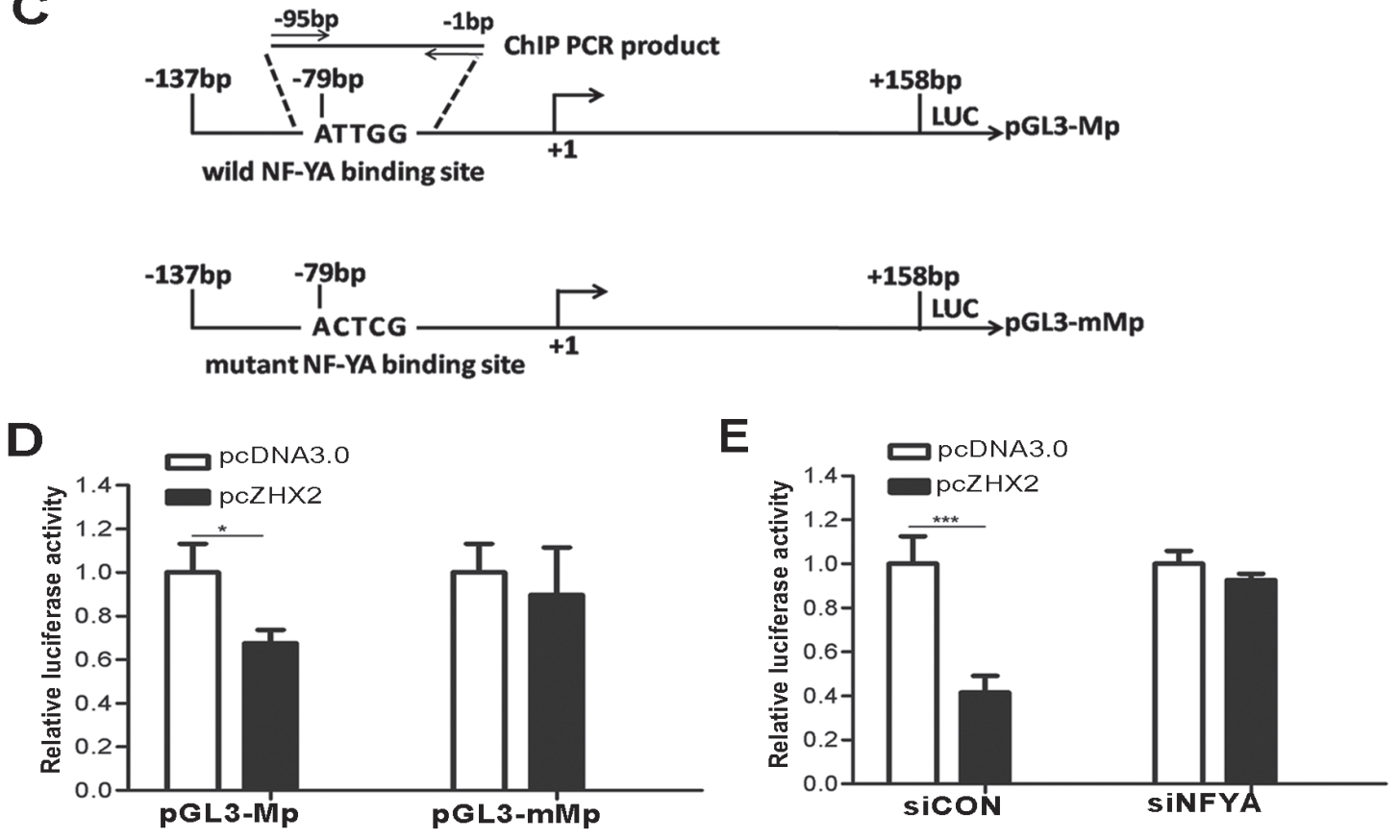

Figure 6: ZHX2 represses $\boldsymbol{M D R} 1$ promoter activity via NF-YA-dependent interactions. (A) HepG2 and HepG2.2.15 cells were co-transfected with pGL3-Mp along with pcDNA3.0 or pcZHX2 (left panel) or with pGL3-Mp and increasing amounts of pcZHX2 (right panel). (B) SMMC7721 cells were transfected with pGL3-Mp and siCON or ZHX2 siRNAs (left panel) or with pGL3-Mp and increasing amounts of $Z H X 2$ siRNAs (right panel).(C) Diagram of the wild type and mutant type MDR1 promoters, showing the location of the $Y$ box and the mutation used to generate pGL3-mMp. (D) HepG2 cells were co-transfected with pGL3-Mp or pGL3-mMp along with pcDNA3.0 or pcZHX2.(E) HepG2 cells were co-transfected with pGL3-Mp and pcDNA3.0 or pcZHX2(Left).In addition, cells were also transfected with siCON or NF-YA siRNA (siNF-YA, Right). Data are shown as the mean $\pm \mathrm{SD}(\mathrm{n} \geq 3) ;{ }^{*} p<0.05,{ }^{* *} p<0.01, * * * p<0.001$. 


\section{DISCUSSION}

It is well established that MDR1/Pgp is a drug efflux pump responsible for the transport of a variety of antineoplastic drugs from the cells. Elevated MDR1 is strongly implicated in MDR and decreases the efficacy of cancer chemotherapy [3, 21]. Inhibition of MDR1/Pgp has been proposed as a powerful way to overcome effluxmediated chemotherapy resistance in cancer cells $[3,4]$.
Here, we identified the ubiquitous transcription factor ZHX2 as a negative regulator of MDR1 transcription. Increased ZHX2 levels led to reduced drug efflux and enhanced the cytotoxicity of anti-cancer drugs in HCC cell lines. Elevated ZHX2 levels significantly enhanced CDDP mediated suppression of liver tumor growth in vivo. These data are consistent with previous clinical studies demonstrating an association between ZHX2 expression and enhanced survival of patients with multiple myeloma

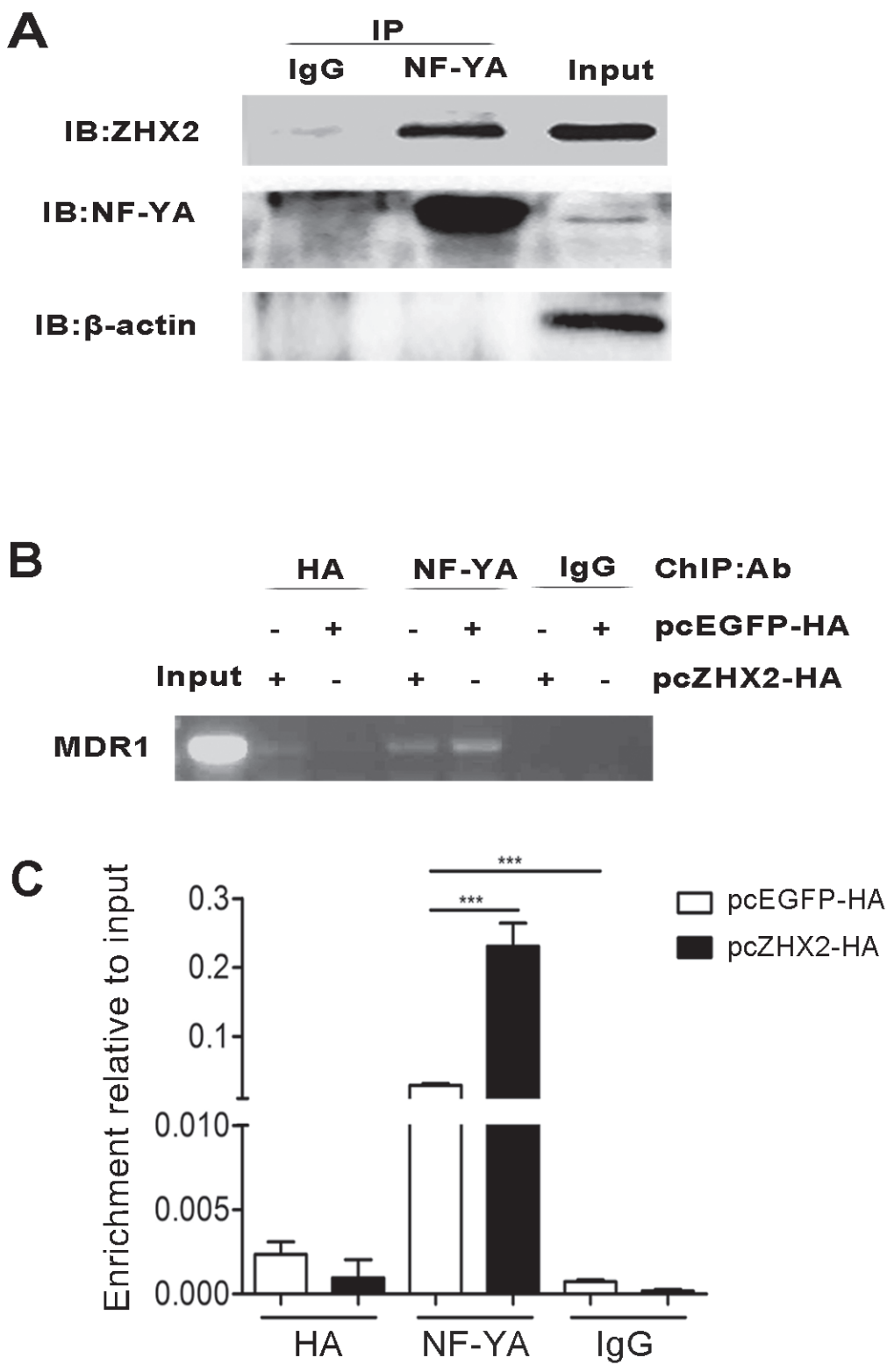

Figure 7: ZHX2 interacts with the MDR1 promoter via NF-Y. (A) Co-IP of NF-YA and ZHX2 after transfection. pcZHX2 plasmid was transfected into HepG2 cells, followed by immunoprecipitations performed as the methods. (B and C) ChIP analysis of DNA from HepG2 cells transfected with pcEGFP-HA or pcZHX2-HA . (B) Conventional PCR amplification of DNA was performed with primers specific to the MDR1 promoter after immunoprecipitation with anti-HA, anti-NF-YA or IgG (control). (C) DNA enrichment was analyzed at the MDR1 promoter by real-time PCR and the results are presented as fold of enrichment over input. Data are shown as the mean $\pm \mathrm{SD}(\mathrm{n}=3) ; * * * p<0.001$. 
after chemotherapy treatment $[17,22]$.

ZHX2 was originally identified based on its interactions with ZHX1 and NF-Y [9]. Subsequently, ZHX2 was found to repress the expression of genes that are frequently upregulated in HCC, including AFP, GPC3 and $\mathrm{H} 19[11,12]$. We recently identified cyclin $\mathrm{A}$ and cyclin E as ZHX2 targets [16]. Here, we added MDR1 as a new target of ZHX2. Based on our results, we propose that ZHX2 represses MDR1 transcription by interacting with NF-YA that is bound to the CCAAT box in the MDRI core promoter region. Previous studies demonstrated that NF-Y was an important regulator of MDR1 expression [6]. Our transient transfections confirmed the importance of the CCAAT box (Figure 6D) and the requirement of NF-Y (Figure 6E) for ZHX2-mediated repression. The co-immunoprecipitation assays confirmed previous reports showing interactions between ZHX2 and NF-YA (Figure 7A) [9]. This interaction between ZHX2 and NFYA on the MDR1 promoter was further verified by ChIP analysis (Figure 7 B and C). Previous studies identified NF-Y as a major component of MDRI transcriptional complex, termed the MDR1 "enhancesome", which is responsible for regulation of MDR1 transcription by a variety of stimuli, such as UV irradiation, HDAC (histone deacetylases) inhibitors and certain chemotherapeutics [23-25]. Moreover, NF-Y is required for recruitment of the histone acetyltransferase $\mathrm{P} / \mathrm{CAF}$ or histone methyltransferase specific for H3K4 MLL1(mixed lineage leukemia 1) to the MDRI promoter, resulting in the transcriptional activation that is likely mediated by further chromatin remodeling [24, 26]. Whether ZHX2 disturb the binding of the enhancesome and the recruitment of chromatin-modifying factors to the $M D R 1$ promoter will require further studies.

Our data presented here demonstrate that ZHX2 inhibits MDR1 expression, which promotes the intracellular accumulation of chemotherapeutic drugs and increases their cytotoxic effects in HCC. This model is supported by ADM accumulation in HepG2 cell (Figure 2C). The combined effect of ZHX2 and CDDP in the inhibition of tumor growth in vivo further supports the hypothesis. CDDP is generally believed to kill cancer cells by binding to DNA and interfering with cellular DNA repair mechanism, which eventually cause apoptosis [27, 28]. As expected, we detected CDDP induced apoptosis in cultured HCC cells and HepG2.2.15 cells grown in nude mice. Although ZHX2 transfer alone did not cause obvious apoptosis, ZHX2 overexpression significantly increased CDDP-induced cell apoptosis both in vitro and in vivo (Figure 4 and 5D). In addition, western blots showed that combination of ZHX2 overexpression and CDDP led to increased release of cytochrome $\mathrm{c}$ and enhanced cleavaged caspase-3, caspase-9 and PARP (Figure 4D). This is consistent with the previous report which demonstrated the CDDP induced mitochondria-driven apoptosis in tumor cells [29]. Although chemotherapeutic agents exert their effects on tumor cells through various mechanisms, apoptosis is likely the final pathway shared by most chemotherapeutic agents [30, 31]. Nagel et al. found that ZHX2 activated STAT1 signaling, which could contribute to apoptosis [15]. This raises the potential that ZHX2 promotes chemotherapy effects by regulating apoptosis related genes and might lead to efflux-independent chemotherapy resistance in HCC cells.

We recently reported that ZHX2 inhibits Cyclin $\mathrm{A}$ and Cyclin E expression, leading to $\mathrm{HCC}$ cell cycle arrest [16]. This indicates that ZHX2 functions as a tumor suppressor in HCC. Our data provided here indicates that ZHX2 represses MDR1 expression and therefore enhances the effect of chemotherapy via increased intracellular drug concentration. This is therefore a second mechanism by which ZHX2 influence HCC cell growth. Further investigation using clinical specimens will be required to determine whether abnormal ZHX2 expression in liver tumors may be used as a potential biomarker for predicting chemotherapeutic drug resistance. Our data also suggest that increasing ZHX2 levels may decrease the growth of HCC cells and increase their sensitivity to chemotherapeutic agents. Identifying additional ZHX2 targets may also elucidate other HCC therapy targets. In conclusion, the results provide a basis for further clinical research in combining ZHX2 and chemotherapeutic agents to treat liver cancer.

\section{MATERIALS AND METHODS}

\section{Cell lines, plasmids and siRNAs}

The human HCC cell lines SMMC7721 were cultured in RPMI 1640. Human hepatoma cell lines HepG2 were cultured in minimum essential medium (MEM) with $1 \mathrm{mmol} / \mathrm{L}$ sodium pyruvate. HepG2.2.15 cells were cultured in MEM with $380 \mu \mathrm{g} / \mathrm{ml} \mathrm{G} 418$ (GIBCO). Human embryonic kidney 293 (HEK293) cells were cultured in Dulbecco's modified Eagle's medium. All the cells were purchased from Shanghai Institute of Cell Biology (Chinese Academy of Sciences ,Shanghai, China), and all the media were supplemented with $10 \%$ fetal bovine serum (FBS, GIBCO).

ZHX2 expression vectors pcZHX2 and pEGFPZHX2 and the siRNAs against ZHX2 (ZHX2-1674, ZHX2-2360) were described previously [16]. pcEGFPHA was constructed by cloning the EGFP-HA to the pcDNA3.0 plasmid. The promoter regions of human MDR1(-137 to +158 , the transcription initiation site designated as +1 ) was cloned into the promoterless pGL3basic vector (Promega) to prepare luciferase reporter plasmid (pGL3-Mp). Reporter plasmid containing mutant MDR1 promoter (pGL3-mMp) was constructed by mutating the wild-type Y-box (ATTGG to ACTCG). The 
siRNA against NF-YA was purchased (sc-2997, Santa Cruz, CA, USA).

\section{RT-PCR and Western blot}

Total RNA extracted with TRIzol reagent (Invitrogen) were reverse transcribed into cDNAs using a Thermo Scientific RevertAid First Strand cDNA Synthesis Kit. Conventional PCR was carried out in a BioRad Thermal Cycler with specific primers (Table S1).

Cytoplasmic extracts and whole cell extracts were prepared as described previously [16]. $40 \mu \mathrm{g}$ of protein separated by SDS-PAGE were bloted with following antibodies: anti-ZHX2 (ab56886, Abcam, MA, USA), anti- $\beta$-actin (sc-1616-R, Santa Cruz, CA, USA), anti-MDR1(sc-55510, Santa Cruz, CA, USA) and anti-cytochrome c (4280), anti-caspase-3 (9668), anticaspase-9 (9508), anti-PARP (9532) from Cell Signal Technology, MA, USA.

\section{Cytotoxicity assay}

To evaluate the effect of ZHX2 on the toxicity of chemotherapeutic drugs, ZHX2-overexpressing cells or ZHX2-knockdown cells were treated with Cisplatin (CDDP, 20 $\mu \mathrm{g} / \mathrm{ml}$; QiLu Pharmaceutical, Jinan, China) or Adriamycin (ADM, $20 \mu \mathrm{g} / \mathrm{ml}$; Pfizer Pharm, NY, USA) for 24 hours. Cell viability was assayed using the Cell Counting Kit-8 (CCK-8, Dojindo, Shanghai, China). The cytotoxicity index was calculated as $\left(1-\mathrm{OD}_{450}\right.$ of drugtreated cells $/ \mathrm{OD}_{450}$ of untreated cells $) \times 100 \%$. The $\mathrm{IC}_{50}$ values of CDDP and ADM were calculated using GraphPad Prism 5 (Version 5.01, GraphPad Software, San Diego, CA) [32].

\section{Cell cycle and apoptosis analysis}

Cells transfected with pcZHX2 or pcDNA3.0 were treated with CDDP $(20 \mu \mathrm{g} / \mathrm{ml})$ for 24 hours. Apoptotic cells were estimated either by Hoechst $33258(1 \mu \mathrm{g} / \mathrm{ml}$, Promega) or DAPI ( $1 \mu \mathrm{g} / \mathrm{ml}$, Promega) staining or flow cytometry with the Apoptosis detection kit (BU-AP0103, Biouniquer Technology Co, Ltd, Nanjing, China). The cell cycle were anlayzed by flow cytometry after propidium iodide (PI, Sigma, USA) staining. Flow cytometery were performed with Beckman Coulter Flow Cytometer (Miami, USA).

\section{Clinical samples and immunohistochemical staining}

To estimate the correlationship of ZHX2 and MDR1 in HCC, ZHX2 and MDR1 immunohistochemical staining was performed in HCC tissues from $30 \mathrm{HCC}$ patients
(Table S2) who underwent surgery between 30 October 2013 and 29 August 2014 at Qilu Hospital and Shandong Provincial Hospital affiliated to Shandong University (Shandong, China). None of the patients was positive for $\mathrm{HCV}$ or HIV. Informed consent was obtained from all patients before the study was initiated with approval of the Shandong University Medical Ethics Committee in accordance with the Declaration of Helsinki.

Immunohistochemical staining using anti-ZHX2 (ab56886, Abcam, MA, USA) and anti-MDR1 (sc55510, Santa Cruz, CA, USA) antibody was performed and analyzed as described previously [16]. Eight fields of $\sim 1000$ cells from each HCC sections were counted independently by three pathologists. Nuclear ZHX2 staining and membrane MDR1 staining were reported separately according to the German semi-quantitative scoring system [33, 34]. Briefly, each sample was scored according to staining intensity (no staining $=0$; weak staining $=1$; moderate staining $=2$; strong staining $=3)$ and the number of stained cells $(0 \%=0 ; 1-25 \%$ $=1 ; 26-50 \%=2 ; 51-75 \%=3 ; 76-100 \%=4)$. Final immunoreactive scores were determined by multiplying the staining intensity by the number of stained cells, with minimum and maximum scores of 0 and 12 , respectively [35].

\section{In vivo xenograft tumor studies}

Male Balb/c nude mice (4 6 weeks of age) were purchased from the Animal Research Committee of Institute of Biology and Cell Biology (Shanghai, China) and housed under specific pathogen-free conditions according to protocols approved by the Shandong University Animal Care Committee. In vivo studies were performed as described previously [16]. Briefly, HepG2.2.15 cells xenografts were prepared and treated by intratumoral injection of pcZHX2 $(20 \mu \mathrm{g})$ or pcDNA3.0 $(20 \mu \mathrm{g})$ together with CDDP $(80 \mu \mathrm{g})$ at 3 days interval for four times. The tumor volume and tumor weight were estimated. Animal experiments(6 mice per group) were repeated at least twice.

\section{TUNEL assays}

Detection of apoptosis in mouse tumor tissues was carried out by terminal deoxyribonucleotidyl transferasemediated dUTP nicked labeling (TUNEL) analysis ,and was performed according to the manufacturer's protocol (In Situ Cell Death Detection Kit, Fluorescein Roche, Cat. No.11684795910 ). 


\section{Intracelluar drug accumulation and retention assay}

The natural red fluorescence of the chemotherapeutic drug ADM was used to determine drug accumulation and retention[36]. Briefly, HepG2 cells transfected with pEGFP-ZHX2 were cultured in medium with $40 \mu \mathrm{g} /$ $\mathrm{ml} \mathrm{ADM}$ for 4 hours at $37^{\circ} \mathrm{C}$. Drug accumulation in the cells was estimated either by fluorescence microscope or flow cytometry. For flow cytometry, cells were gated as EGFP-positive and EGFP-negative, then red MFI of the gated cells was used to determine drug accumulation. To measure the drug retention, ADM treated cells were cultured with fresh, drug-free medium for another 2 hours allowing drug efflux from cells. The ADM releasing index of HepG2 cells was calculated as following: releasing index $=($ accumulation MFI-retention MFI)/accumulation MFI.

\section{Co-immunoprecipitation(Co-IP)}

Cell lysates from HepG2 cells transfected with pcZHX2 were precipitated with control IgG (sc-2027, Santa Cruz, CA, USA) or NF-YA antibody (ab6558; Abcam, Hong Kong). After incubation with protein A/G Plus-Agarose(sc-2003, Santa Cruz, CA, USA), the precipitates were immunobloted with anti-HA antibody (ab9110; Abcam).

\section{Chromatin immunoprecipitation(ChIP) assays}

ChIP assays was performed with HepG2 cells transfected with pcZHX2-HA or pcEGFP-HA as reported [16]. Briefly, fixed cells were sonicated to shear DNA to $200 \sim 1000 \mathrm{bp}$ and immunoprecipitated using anti-HA antibody (ab9110; Abcam), anti-NF-YA antibody (ab6558; Abcam) or rabbit IgG (sc-2027, Santa Cruz, CA, USA). As controls, 1/20th of the starting chromatin (Input) was used. Analysis was performed using specific primers for the NF-YA binding region of $M D R 1$ promoter.

\section{Real-time quantitative PCR}

Real-time quantitative PCR was carried out in BioRad C1000 ${ }^{\mathrm{TM}}$ Thermal Cycler CFX96 ${ }^{\mathrm{TM}}$ RealTime System using SuperReal PreMix Plus (SYBR Green,TIANGEN BIOTECH,BEIJING). The results are presented as fold of enrichment over input. The used primers were shown in Table S1.

\section{Luciferase reporter assays}

HCC cell lines were transfected with reporter

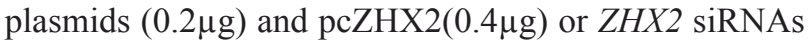
using Lipofectamine ${ }^{\mathrm{TM}}$ 2000(Invitrogen). To further explore the role of NF-YA in ZHX2 mediated control of the MDR1 promoter, HepG2 cells were co-transfected with reporter plasmids, $N F-Y A$ siRNA and pcZHX2. Luciferase reporter assay was performed using Dual-Luciferase Reporter Assay System (Promega).

\section{Statistics}

GraphPad Prism (GraphPad Software, San Diego, CA) was used for data analysis. Data values were presented as the means $\pm \mathrm{SD}$. The statistical correlation between the MDR1 staining levels and the ZHX2 staining levels in tissue sections was analyzed by the Chi-square test. Two-way ANOVA followed by Bonferroni posttests was applied to determine significant differences between different treatments in xenograft tumor studies. The Student t-test was applied to determine significant differences between groups. In these analyses, $p<0.05$ were considered to statistically significant.

\section{ACKNOWLEDGEMENTS}

We would like to thank Limei Wang for excellent technical support (Shandong University School of Medicine). This work was supported in part by grants from the NSFC of China (81370527 and 30972753), the Programme for NCET-10-0524, and the The National Natural Science Fund for Outstanding Youth Fund (81425012).

\section{Conflict of interest}

The authors declare that they have no conflict of interest.

\section{Abbreviations}

ZHX2, Zinc-fingers and homeoboxes 2; HCC, hepatocellular carcinoma; MDR, multidrug resistance; CDDP, cis-diamminedichloroplatinum; ADM, Adriamycin.

\section{REFERENCES}

1. Asghar $U$ and Meyer T. Are there opportunities for chemotherapy in the treatment of hepatocellular cancer? J Hepatol. 2012;56:686-695.

2. Hernandez-Gea V, Toffanin S, Friedman SL and Llovet 
JM. Role of the microenvironment in the pathogenesis and treatment of hepatocellular carcinoma. Gastroenterology. 2013;144:512-527.

3. Tsuruo T. Molecular cancer therapeutics: recent progress and targets in drug resistance. Intern Med. 2003;42:237243.

4. Park JG, Lee SK, Hong IG, Kim HS, Lim KH, Choe KJ, Kim WH, Kim YI, Tsuruo T and Gottesman MM. MDR1 gene expression: its effect on drug resistance to doxorubicin in human hepatocellular carcinoma cell lines. J Natl Cancer Inst. 1994;86:700-705.

5. Cheng SC, Zhou J and Xie Y. P-glycoprotein expression induced by glucose depletion enhanced the chemosensitivity in human hepatocellular carcinoma cell-lines. Cell Biol Int. 2005;29:269-275.

6. Fang D, Guo Y, Zhu Z and Chen W. Silence of p15 expression by RNAi enhances cisplatin resistance in hepatocellular carcinoma cells. Bosn J Basic Med Sci. 2012;12:4-9.

7. Szakacs G, Paterson JK, Ludwig JA, Booth-Genthe C and Gottesman MM. Targeting multidrug resistance in cancer. Nat Rev Drug Discov. 2006;5:219-234.

8. Goldsmith ME, Madden MJ, Morrow CS and Cowan KH. A $\mathrm{Y}$-box consensus sequence is required for basal expression of the human multidrug resistance (mdr1) gene. J Biol Chem. 1993;268:5856-5860.

9. Sundseth R, MacDonald G, Ting J and King AC. DNA elements recognizing NF-Y and $\mathrm{Sp} 1$ regulate the human multidrug-resistance gene promoter. Mol Pharmacol. 1997;51:963-971.

10. Dorn A, Bollekens J, Staub A, Benoist C and Mathis D. A multiplicity of CCAAT box-binding proteins. Cell. 1987;50:863-872.

11. Spear BT, Jin L, Ramasamy S and Dobierzewska A. Transcriptional control in the mammalian liver: liver development, perinatal repression, and zonal gene regulation. Cell Mol Life Sci. 2006;63:2922-2938.

12. Kawata H, Yamada K, Shou Z, Mizutani T, Yazawa T, Yoshino M, Sekiguchi T, Kajitani T and Miyamoto K. Zinc-fingers and homeoboxes (ZHX) 2, a novel member of the ZHX family, functions as a transcriptional repressor. Biochem J. 2003;373:747-757.

13. Yamada K, Ogata-Kawata H, Matsuura K, Kagawa N, Takagi K, Asano K, Haneishi A and Miyamoto K. ZHX2 and ZHX3 repress cancer markers in normal hepatocytes. Front Biosci (Landmark Ed). 2009;14:3724-3732.

14. Morford LA, Davis C, Jin L, Dobierzewska A, Peterson ML and Spear BT. The oncofetal gene glypican 3 is regulated in the postnatal liver by zinc fingers and homeoboxes 2 and in the regenerating liver by alpha-fetoprotein regulator 2 . Hepatology. 2007;46:1541-1547.

15. Perincheri S, Dingle RW, Peterson ML and Spear BT. Hereditary persistence of alpha-fetoprotein and H19 expression in liver of $\mathrm{BALB} / \mathrm{cJ}$ mice is due to a retrovirus insertion in the Zhx2 gene. Proc Natl Acad Sci U S A. 2005;102:396-401.

16. Yue X, Zhang Z, Liang X, Gao L, Zhang X, Zhao D, Liu X, Ma H, Guo M, Spear BT, Gong Y and Ma C. Zinc fingers and homeoboxes 2 inhibits hepatocellular carcinoma cell proliferation and represses expression of Cyclins A and E. Gastroenterology. 2012;142:1559-1570 e1552.

17. Armellini A, Sarasquete ME, Garcia-Sanz R, Chillon MC, Balanzategui A, Alcoceba M, Fuertes M, Lopez R, Hernandez JM, Fernandez-Calvo J, Sierra M, Megido M, Orfao A et al. Low expression of ZHX2, but not RCBTB2 or RAN, is associated with poor outcome in multiple myeloma. Br J Haematol. 2008;141:212-215.

18. Nagel S, Schneider B, Rosenwald A, Meyer C, Kaufmann M, Drexler HG and MacLeod RA. $\mathrm{t}(4 ; 8)(\mathrm{q} 27 ; \mathrm{q} 24)$ in Hodgkin lymphoma cells targets phosphodiesterase PDE5A and homeobox gene ZHX2. Genes Chromosomes Cancer. 2011;50:996-1009.

19. Nagel S, Schneider B, Meyer C, Kaufmann M, Drexler HG and Macleod RA. Transcriptional deregulation of homeobox gene ZHX2 in Hodgkin lymphoma. Leuk Res. 2012;36:646-655.

20. Chu G. Cellular responses to cisplatin. The roles of DNA-binding proteins and DNA repair. J Biol Chem. 1994;269:787-790.

21. Gottesman MM, Fojo T and Bates SE. Multidrug resistance in cancer: role of ATP-dependent transporters. Nat Rev Cancer. 2002;2:48-58.

22. Legartova S, Harnicarova-Horakova A, Bartova E, Hajek R, Pour L and Kozubek S. Expression of RAN, ZHX2, and $\mathrm{CHC1L}$ genes in multiple myeloma patients and in myeloma cell lines treated with HDAC and Dnmts inhibitors. Neoplasma. 2010;57:482-487.

23. Scotto KW. Transcriptional regulation of $\mathrm{ABC}$ drug transporters. Oncogene. 2003;22:7496-7511.

24. Jin S and Scotto KW. Transcriptional regulation of the MDR1 gene by histone acetyltransferase and deacetylase is mediated by NF-Y. Mol Cell Biol. 1998;18:4377-4384.

25. Hu Z, Jin S and Scotto KW. Transcriptional activation of the MDR1 gene by UV irradiation. Role of NF-Y and Sp1. J Biol Chem. 2000;275:2979-2985.

26. Huo H, Magro PG, Pietsch EC, Patel BB and Scotto KW. Histone methyltransferase MLL1 regulates MDR1 transcription and chemoresistance. Cancer Res. 2010;70:8726-8735.

27. Rosenberg B, Vancamp L and Krigas T. Inhibition of Cell Division in Escherichia Coli by Electrolysis Products from a Platinum Electrode. Nature. 1965;205:698-699.

28. Wang D and Lippard SJ. Cellular processing of platinum anticancer drugs. Nat Rev Drug Discov. 2005;4:307-320.

29. Azuma M, Tamatani T, Ashida Y, Takashima R, Harada K and Sato M. Cisplatin induces apoptosis in oral squamous carcinoma cells by the mitochondria-mediated but not the NF-kappaB-suppressed pathway. Oral Oncol. 2003;39:282- 
289.

30. Stege A, Priebsch A, Nieth $\mathrm{C}$ and Lage H. Stable and complete overcoming of MDR1/P-glycoprotein-mediated multidrug resistance in human gastric carcinoma cells by RNA interference. Cancer Gene Ther. 2004;11:699-706.

31. Xu D, McCarty D, Fernandes A, Fisher M, Samulski RJ and Juliano RL. Delivery of MDR1 small interfering RNA by self-complementary recombinant adeno-associated virus vector. Mol Ther. 2005;11:523-530.

32. Hanapi NA, Ismail S and Mansor SM. Inhibitory effect of mitragynine on human cytochrome P450 enzyme activities. Pharmacognosy Res. 2013;5:241-246.

33. Han CP, Kok LF, Wang PH, Wu TS, Tyan YS, Cheng YW, Lee MY and Yang SF. Scoring of p16(INK4a) immunohistochemistry based on independent nuclear staining alone can sufficiently distinguish between endocervical and endometrial adenocarcinomas in a tissue microarray study. Mod Pathol. 2009;22:797-806.

34. Kamoi S, AlJuboury MI, Akin MR and Silverberg SG. Immunohistochemical staining in the distinction between primary endometrial and endocervical adenocarcinomas: Another viewpoint. International Journal of Gynecological Pathology. 2002;21:217-223.

35. Han CP, Lee MY, Tzeng SL, Yao CC, Wang PH, Cheng YW, Chen SL, Wu TS, Tyan YS and Kok LF. Nuclear Receptor Interaction Protein (NRIP) expression assay using human tissue microarray and immunohistochemistry technology confirming nuclear localization. J Exp Clin Cancer Res. 2008;27:25.

36. Zhang YF, Li XH, Shi YQ, Wu YY, Li N, He QA, Ji Q, Wang RQ, Yang SM and Fang DC. CIAPIN1 confers multidrug resistance through up-regulation of MDR-1 and Bcl-L in LoVo/Adr cells and is independent of p53. Oncology Reports. 2011;25:1091-1098. 